\title{
Analyzing and evaluation of vintage logs for an integrated reservoir characterization and field development strategy
}

Abdulmalik Adetunji Taj-Liad

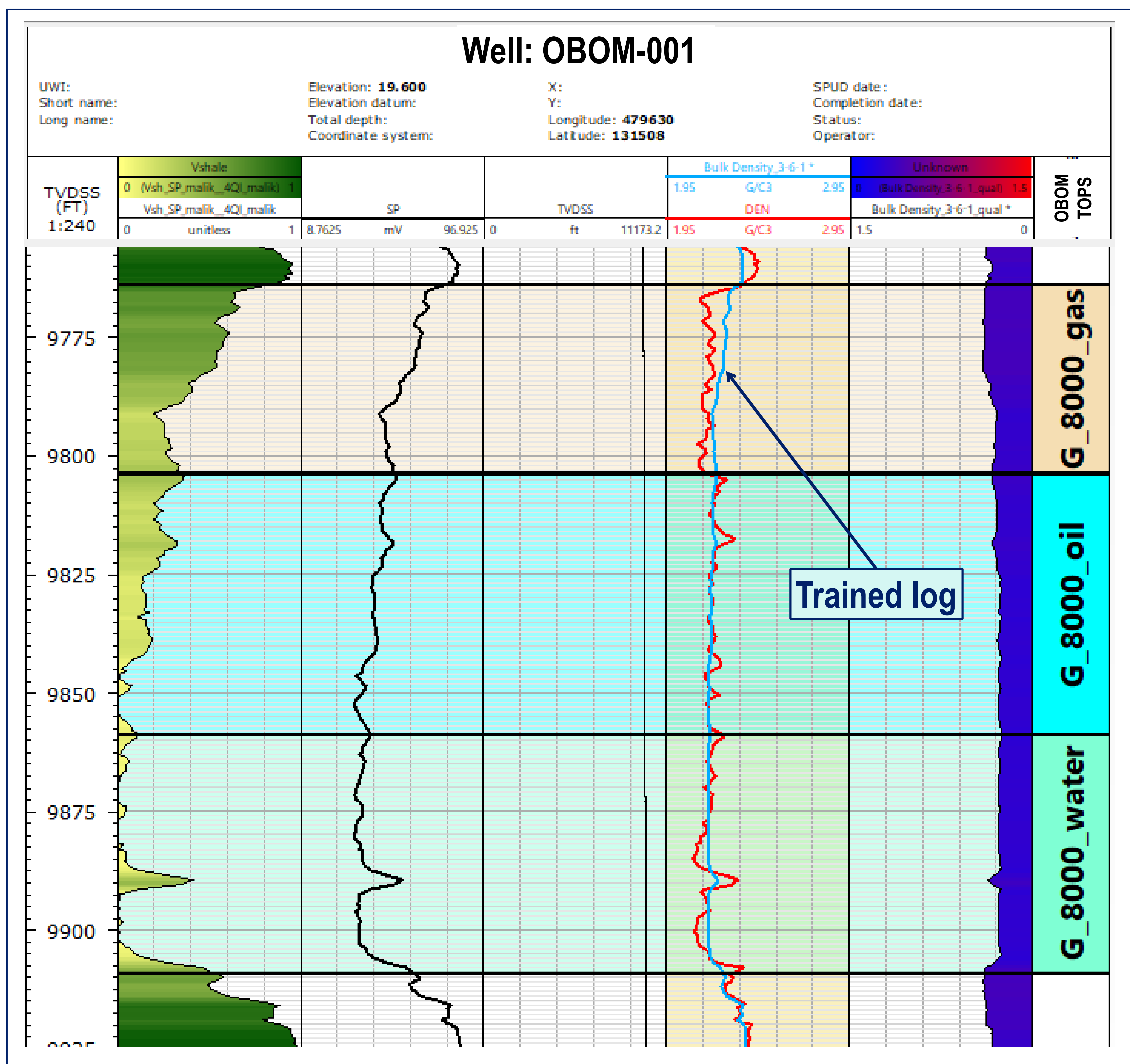

\section{INTRODUCTION}

Obom field is a mature field in the Greater Ughelli onshore Niger delta, which has been producing since 1967. The field is a simple rollover structure elongated in the east-west direction, and bounded to the north by an east-west trending, south-hading, main growth fault. The reservoirs are made mainly of channel/shoreface complexes. The closures are faults assisted dip closures in shallow reservoir and dip assisted fault closure in deeper sections. As a huge producing field with some potential for further sustainable production, field monitoring is therefore important in the identification of areas of unproduced hydrocarbon. The aim of this study is to train logs to get pseudo logs to evaluate them alongside acquired logs which will serve as an input into other disciplines for an integrated field development study.

\section{RESULTS}

- Density logs were trained from supervised neural network (K.mod - using Vshale, SP logs and Tvdss) for both radioactive and non-radioactive levels. The trained density logs gave a better well-to-seismic tie for the synthetic seismogram generation compared to linear regression.

- Volume of shale was evaluated from both gamma ray (GR) and spontaneous potential (SP) log (due to radioactive level and paucity of neutron/density logs) and spliced with data editor to give a final volume of shale.

- The use of python scripting saved computing time by more than $70 \%$ due to the numbers of wells in the field - fourteen wells. Permeability evaluation and hydrocarbon correction on porosity were all done with python scripts.

\section{CONCLUSION}

Using neural network (Techlog k.mod) offers a more robust workflow to get pseudo logs thus, gives a closer representation to acquired logs compared to a linear regression.

Techlog Python helps to save computing time when evaluation of logs has to be done on multiple wells.

This study has demonstrated the effectiveness of integrating trained dataset for field development study while using python scripts to save computing time. Hence, providing a framework for future prediction of reservoir performance and production behavior of the field.
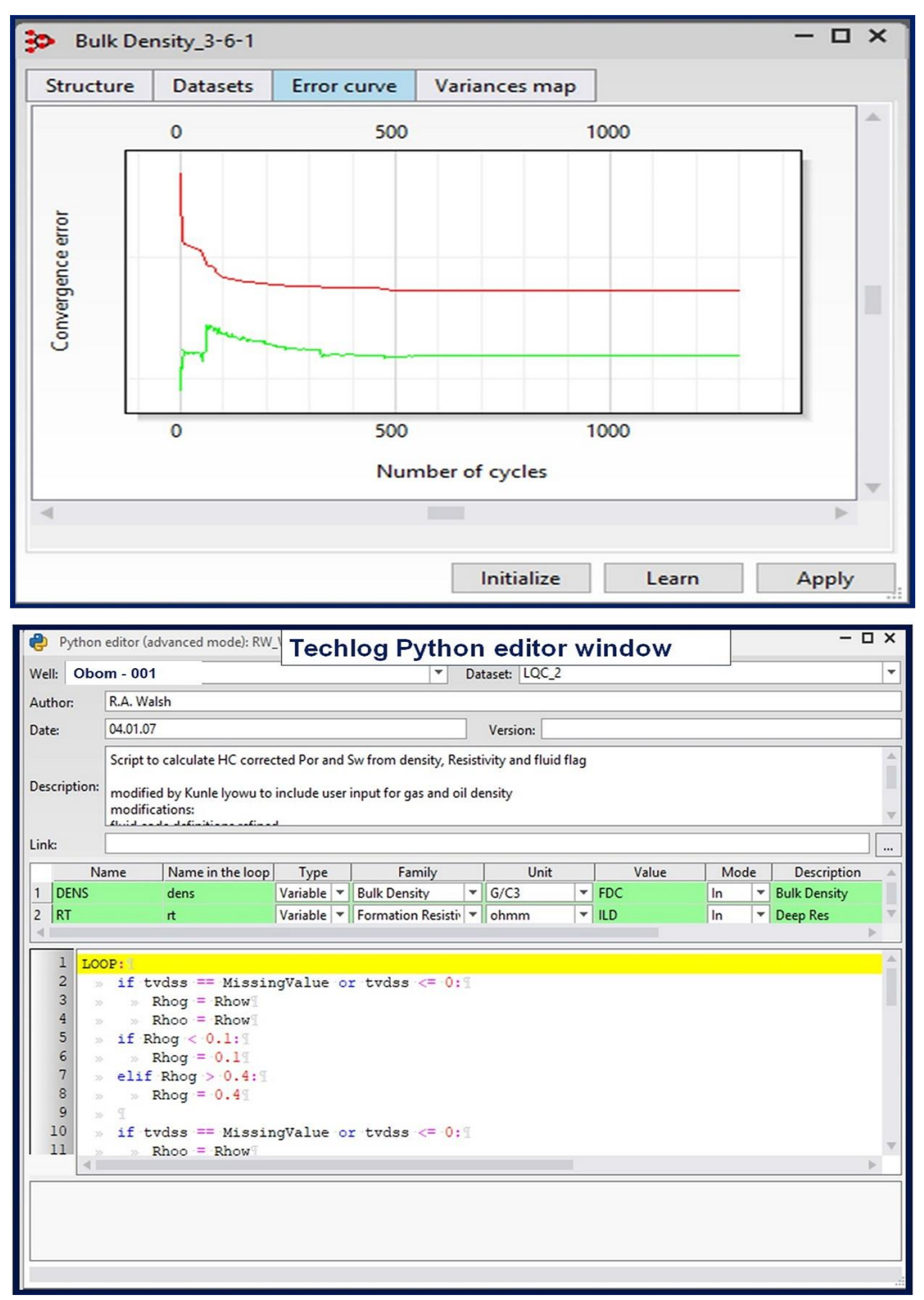